\title{
Automatic Centerline Extraction and Error Analysis Based on Machine Vision for Bending Pipe Image
}

\author{
Hui Wen ${ }^{1, a}$, Duanling $\mathrm{Li}^{2, \mathrm{~b}}$ and Jinquan $\mathrm{Li}^{3, \mathrm{C}}$ \\ ${ }^{1}$ Automation School of Beijing University of Posts and Telecommunications, Beijing \\ ${ }^{2}$ Automation School of Beijing University of Posts and Telecommunications, Beijing \\ ${ }^{3}$ Automation School of Beijing University of Posts and Telecommunications, Beijing \\ asmart1201@163.com, ${ }^{\mathrm{b}}$ liduanling@126.com clijinquan@bupt.edu.cn
}

Keywords: Machine vision, pipe centerline, perspective error, error compensation.

\begin{abstract}
Centerline reflects the shape characteristics of pipes, and centerline extraction is widely used in automation equipment field and Medical field. This paper proposes a method to automatically extract bending pipe's centerline with technology of image filtering and de-noising and edge detection. By finding the outer edge of the pipe, the paper creates a distance model, and the centerline of the pipe is a series of points with shortest distance between two outside edges. In error analysis, the paper analyzes the radial distortion and tangential distortion caused by a camera and also analyzes the perspective error which is very important to centerline extraction problem. By simultaneously analyzing the error of these three areas this paper presents a more accurate calculation method of the centerline. Finally, the paper verifies the correctness of the methods through experiments.
\end{abstract}

\section{Introduction}

Centerline reflect the shape characteristics of pipes, which has a wide range of applications. In the field of automation equipment, it can be used to obtain the position of the metal pipe to complete equipment's polishing and assembly. Diseases can be analyzed by centerline of the vessel from angiographic image obtained by $x$-ray contrast media[1-4]. At present, domestic and foreign scholars have proposed a number of centerline acquisition algorithm. Two mainstream algorithm are raster method and vector method. Raster algorithm is computationally intensive, and this method usually requires the use of tracking algorithm which is complicated to obtain a vector forming the centerline. Vector method generally has a high efficiency, but the tightness is poor, and the accuracy of the results is often poor or is not satisfactory to the curve with complex shape.

If we process the image before extracting the centerline, the extraction process would be greatly simplified. A lot of noise can be removed by using image processing algorithms. However, the image processing has inherent errors. So the paper extracts centerline of pipes using image processing technology, and then analyzes and compensates error.

\section{Centerline Extraction with Image Progressing}

Firstly, filter and morphological processing and neighborhood operations are used to pre-progress the bending pipe image before detecting the edges of the pipe. Two edges of the pipe are orderly stored in two containers. Then we calculate the minimum distance of points of the two containers. Computational complexity is reduced by segment search. Finally, the centerline of the pipe is a series of points with shortest distance between two outside edges.

Geting the Contour of the Pipe. The contour is obtained by edge processing. In this paper, algorithm is discuss on the image of a $19 \mathrm{~mm}$ diameter stainless steel tube, as shown in Fig. 1 . We use the background lighting to get better contour. As can be seen from Fig.1, there are some miscellaneous points on pipes which can be removed by the median filter. Furthermore, the edge of the pipe is blur due to reflection and will be incompletely missing after image binarization which 
can be solved by morphological filtering. Finally, we get the border of the pipe through Canny filtering. The result is showing in Figure 1.
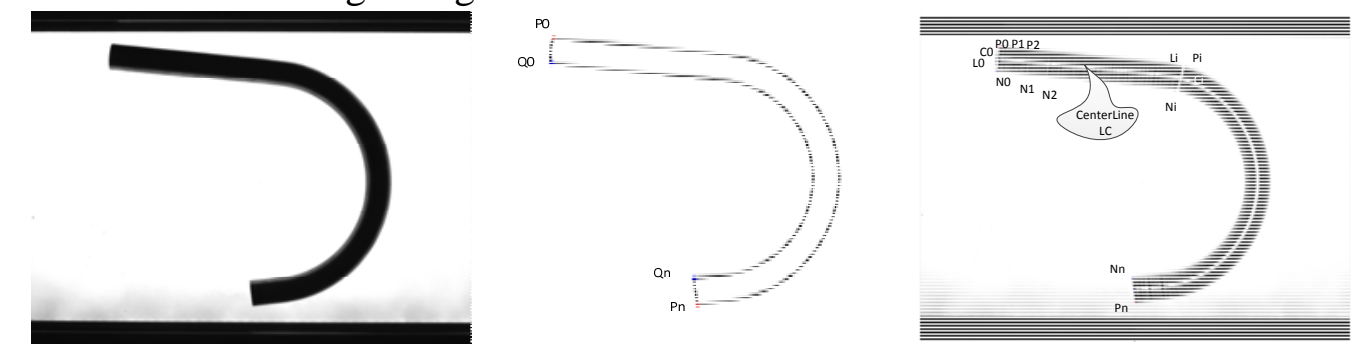

Figure 1. original image and image after Canny filtering and image with centerline

Four head and tail turning points of the pipe can be found by calculating the curvature of the curve, assuming they are the $P_{0}, Q_{0}, P_{n}, Q_{n}$, as is shown in Figure 1. The contour is divided into inner and outer one after erasing the $P_{0} Q_{0}$ and $P_{n} Q_{n}$.

The inner and out contours are separately stored point by point by finding connected points from 4 neighborhood or 8 neighborhood.

Search Points with Minimum Distance between Inner and Out Contours. We search outer contour points' nearest point from inner contour points. For example, when finding the nearest point of $P_{0}$ (Fig.1) from inner contour points, we calculate the Euclidean distance among point $P_{0}$ and all points of inner contour:

Dist $=\operatorname{sqrt}\left(\left(x-P_{0} \cdot x\right)^{2}+\left(y-P_{0} \cdot y\right)^{2}\right)$.

where $(x, y)$ is the inner contour point, if the nearest point is $N_{0}$. Midpoint of point $P_{0}$ and point $N_{0}$ can be found easily, assuming is $C_{0}$ in Fig.1. By the same token we can find the nearest point $N_{i}$ from inner contour of any point $P_{i}$ from outer contour and $C_{i}$ which forms the centerline. And the centerline is the white line showing in Fig.1.

In fact, there is no need to calculate the distance among point $P_{i}$ and all inner points. Search can be simplified by limiting the maximum searching distance according to the width of the pipe or according to the minimum distance of last search. In addition, point $N_{i+1}$ which is the next point of $N_{i}$ should be around $N_{i}$, and computational complexity is reduced by limiting the search position.

Error Caused by Inequality of Curvatures of the Inner and Outer Contours . When there is large curvature difference between the inner and outer contour, the search error will increase. As shown in Figure 2, the nearest point of point $A$ from outer contour can be point $N_{1}$ or point $N_{3}$, and the centerline $l$ is the connection of $\mathrm{C} 1$ and $\mathrm{C} 2$ which are the midpoints of segment $A N_{1}$ and $A N_{3}$. However, the nearest point of point $A$ from outer contour is hope to be point $N_{2}$, and there will be great difference between true centerline and $l$.

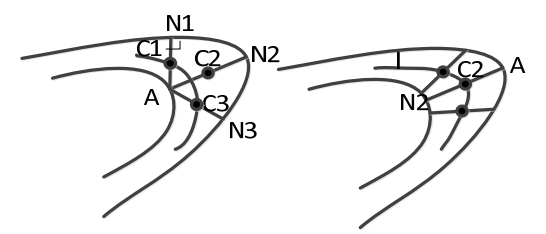

Figure 2 Image with large curvature difference between the inner and outer contour

To address the problem, when there is a big difference between curvatures of inner and outer contour, we find the nearest point of bigger curvature contour points from smaller curvature contour points, not find the nearest point of smaller curvature contour points from bigger curvature contour points. As is shown in Figure 2, we find the nearest point of outer contour from inner contour, not find the nearest point of inner contour from outer contour. 


\section{Error and Error Compensation}

The main error of centerline extraction of bending pipes is image distortion and perspective error.

Error of Perspective. There is no perspective error only when the horizontal cross section of the pipe is parallel to camera imaging plane and the camera is located just above the pipe. However, the camera can't be located above all parts of the pipe because the pipe is much bigger than camera imaging plane. so there is always perspective error.
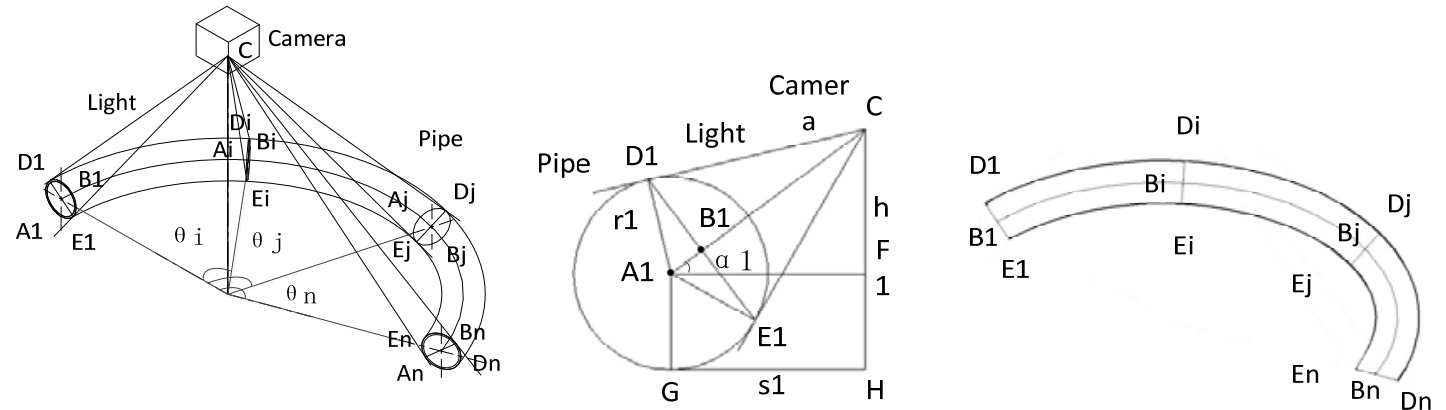

Figure 3 Fluoroscopic imaging model of pipe and centerline image ignoring perspective error

As is shown in Figure 3, the camera is simplified to a point C. Light around the edge of the pipe is tangent to the cross-section circle of pipe from pipe to camera. Supposing the tangent points are the point $D_{1}$ and $E_{1}$, Light is presented by line $D_{1} C$ and line $E_{1} C$. A series of $D_{i}$ and $E_{i}$ can be found after analyzing to the entire cross-section circle. $D_{1}, D_{2}, \ldots, D_{n}$ will be the outer border of pipe and $E_{1} 、 E_{2}, \ldots, E_{n}$ will be the inner border of pipe in the image taken by the camera. Supposing $B_{i}$ is the midpoint of $D_{i}$ and $E_{i}$, and a series of center points $B_{1} 、 B_{2} 、 B_{3}, B_{4}, \ldots, B_{n}$ which form the centerline will be found.

However, centerline $B_{1} B_{2} B_{3} B_{4} \ldots B_{n}$ which is directly obtained by dealing with the image without considering perspective error is not the true centerline of the pipe because line $D_{i} E_{i}$ does not pass through the center of the cross-section circle. As is shown in Figure 3, assuming the center of the cross-section circle is $A_{1} 、 A_{2} 、 A_{3}, \ldots, A_{n}$, the true centerline of pipe is $A_{1} A_{2} A_{3} \ldots A_{n}$.

Error vector can be expressed as $n=\left\{A_{1} B_{1}, A_{2} B_{2}, \ldots, A_{n} B_{n}\right\}$. As is shown in Figure 3 , error is analyzed on first cross-section circle and error on other cross-section circle will be similarly available. As we can see, $\mathrm{CH}$ is the height between camera and pipe and its value is $h, G H$ is the level distance between camera and pipe and its value is $s_{1}, r_{1}$ is the radius of the pipe, and $B_{1}$ is the midpoint of $D_{1} E_{1}$. Obviously $A_{1} C$ is perpendicular to $D_{1} E_{1}$ and $\triangle A_{1} E_{1} C \backsim \Delta A_{1} B_{1} E_{1}$, So the following conclusions can be easily deduced:

$$
A_{1} B_{1}=\frac{A_{1} E_{1}^{2}}{A_{1} C}=\frac{r_{1}^{2}}{\sqrt{s_{1}^{2}+\left(h-r_{1}\right)^{2}}}
$$

Error of $\mathrm{x}$-axis and $\mathrm{y}$-axis and $\mathrm{z}$-axis can be obtained by simply projecting $\mathrm{AB}$ to the three axes:

$$
\begin{aligned}
& \Delta x_{1}=A_{1} B_{1} \times \cos \left(\alpha_{1}\right) \times \cos \left(\theta_{1}\right)=\frac{r_{1}^{2}}{\sqrt{s_{1}^{2}+\left(h-r_{1}\right)^{2}}} \times \cos \left(\alpha_{1}\right) \times \cos \left(\theta_{1}\right) \\
& \Delta y_{1}=A_{1} B_{1} \times \cos \left(\alpha_{1}\right) \times \sin \left(\theta_{1}\right)=\frac{r_{1}^{2}}{\sqrt{s_{1}^{2}+\left(h-r_{1}\right)^{2}}} \times \cos \left(\alpha_{1}\right) \times \sin \left(\theta_{1}\right) \\
& \Delta z_{1}=A_{1} B_{1} \times \sin \left(\alpha_{1}\right)=\frac{r_{1}^{2}}{\sqrt{s_{1}^{2}+\left(h-r_{1}\right)^{2}}} \times \sin \left(\alpha_{1}\right)
\end{aligned}
$$

Where $\theta_{1}$ is the cross-section declination of pipe whose value is determined by the position of point $B_{1}$ and point $H$, and $\theta_{1}=0$, as is shown in Figure 3. $\alpha_{1}$ is the dip angle of $A_{1} B_{1}$ which is 
determined by $B_{1}$ and $C$ and $H$, as shown in Fig. 3.2. So the following conclusions can be easily deduced:

$$
s_{1}=A_{1} F_{1}=\frac{C F_{1}}{\tan \alpha_{1}}=\frac{h-r_{1}}{\tan \alpha_{1}}
$$

The same method can be used to solve $\left(\Delta x_{2}, \Delta y_{2}, \Delta z_{2}\right),\left(\Delta x_{3}, \Delta y_{3}, \Delta z_{3}\right),\left(\Delta x_{3}, \Delta y_{3}, \Delta z_{3}\right), \ldots,\left(\Delta x_{n}, \Delta y_{n}, \Delta z_{n}\right)$. Some conclusions can be drawn by the calculation formula of Error vector: The error (1) increases with the increase of the radius $r$. (2)and decreases with the increase of the height $h$ between the pipe and the camera. (3)and decreases with the increase of the horizontal distance $s$.

Image Distortion Error. Section3.1 analyzes the perspective error because the camera is not located directly above the object; there is another kind of image distortion since the image taking lenses are not perfectly parallel to the imaging plane or due to the "barrel" or "fish-eye" effect, For the distortion we takes into account the radial and tangential factors. For the radial factor one uses the following formula[5]:

$$
\begin{aligned}
& x_{\text {corrected }}=x\left(1+k_{1} r^{2}+k_{2} r^{4}+k_{3} r^{6}\right) \\
& y_{\text {corrected }}=y\left(1+k_{1} r^{2}+k_{2} r^{4}+k_{3} r^{6}\right)
\end{aligned}
$$

For the tangential factor one uses the following formula[5]:

$$
\begin{aligned}
& x_{\text {corrected }}=x+\left[2 p_{1} x y+p_{2}\left(r^{2}+2 x^{2}\right)\right] \\
& y_{\text {corrected }}=y+\left[p_{1}\left(r^{2}+2 y^{2}\right)+2 p_{2} x y\right]
\end{aligned}
$$

So for an old pixel point at (x,y) coordinates in the input image, its position on the corrected output image will be $\left(x_{\text {corrected }}, y_{\text {corrected }}\right)$.

Error Compensation. Compensation for image distortion and perspective error is finished on different stages. Image distortion is corrected firstly so that the pixels are on corrected position, and then two-dimensional pixels are projected to 3D space. Finally, perspective error is corrected as described in section 3.1.Suppose tube radius $r$ is a known quantity. Error is compensated for the by the following procedure:

First, camera is calibrated to obtain the intrinsic parameters of the camera, such as radial distortion factor $k_{1}, k_{2}, k_{3}, k_{4}, k_{5}, k_{6}$ and tangential distortion factor $p_{1}, p_{2}$ and the principal point $\left(c_{x}, c_{y}\right)$. And object distance $h$ is also measured[6].

Then, the pixel coordinate is transformed into space coordinate. That is, we will convert pixel coordinate $\left(u_{i}, v_{i}\right)$ of point $B_{i}$ to space coordinate $\left(\mathrm{X}_{i}, \mathrm{Y}_{i}, Z_{i}\right)$. This progress is calculated by formula follows:

$$
\left[\begin{array}{l}
x \\
y \\
z
\end{array}\right]=R\left[\begin{array}{l}
X \\
Y \\
Z
\end{array}\right]+t
$$

Where $R$ is the rotation vector, $t$ is the translation vector. 3D coordinate point $(\mathrm{X}, \mathrm{Y}, \mathrm{Z})$ will be converted to 2D camera coordinate point $(\mathrm{x}, \mathrm{y})$ through the above formula. The opposite conversion, can be realized by Newton's iterative method. However $(x, y)$ is not the correct output due to image distortion. (x,y) is converted to the corrected location through by the formula in section 3.2.

Finally, perspective error $\left(\Delta x_{i}, \Delta y_{i}, \Delta z_{i}\right)$ of every point $\left(\mathrm{X}_{i}, \mathrm{Y}_{i}, \mathrm{Z}_{i}\right)$ is calculated as described in 3.1:

$$
\begin{aligned}
& \mathrm{X}_{i}=\mathrm{X}_{i}+\Delta x_{i} \\
& \mathrm{Y}_{i}=\mathrm{Y}_{i}+\Delta y_{i} \\
& \mathrm{Z}_{i}=\mathrm{Z}_{i}+\Delta \mathrm{z}_{i}
\end{aligned}
$$

After compensation of image distortion errors and errors perspective, we get the real position of the centerline, i.e., the centerline formed by $A_{1}, A_{2}, \cdots, A_{n}$ in Figure 3. 


\section{Experiment}

Experiments carried out on 500 pipe images by comparison centerline computed by our method and the true centerline position obtained by mechanical measuring instruments. Camera is MER-500-7UC/UM(-L) from Daheng image with a resolution of $2592 \times 1944$. Experiment is performed on stainless steel tube whose diameter ranging from 5 to $50 \mathrm{~mm}$, and the Program is written in VS2010. Experimental procedure is as follows:

(1) camera Calibration;

(2) Take pictures of stainless steel tube;

(3) Get centerline;

(4) Error Compensation.

Part of experimental results are as follows. original pipe is on the left right and the right is pipe with centerline found by our method.

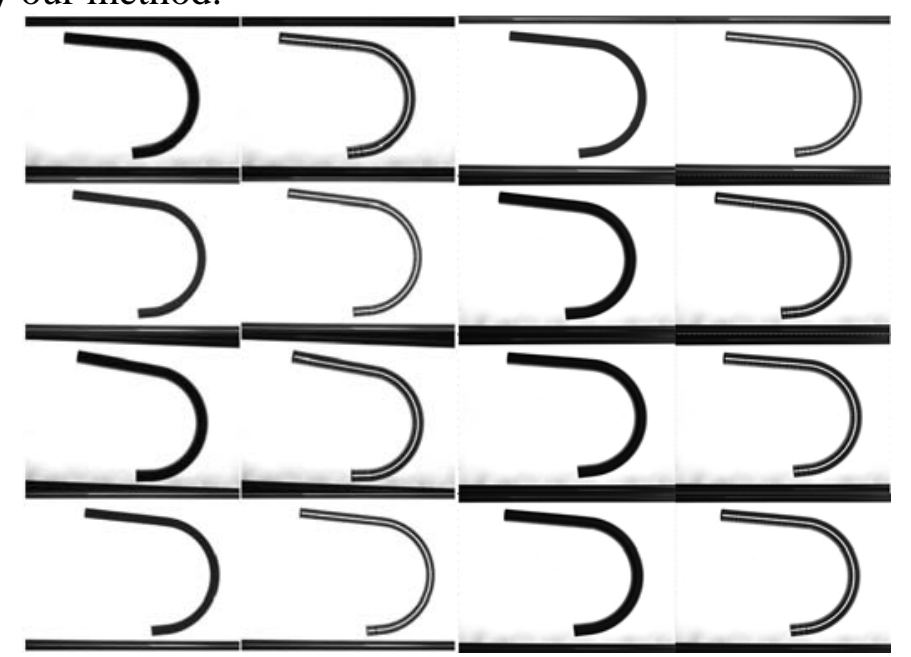

Figure 4 Centerline after error compensation

Experimental results show our method can perform well in different situations and perspective error and image distortion error are compensated effectively. As a result of image processing, tracing algorithm is omitted, which lead directly to the decrease of computational complexity.

\section{Conclusion}

The paper proposes an effective method to calculate the centerline of bending pipes. The method accurately obtains the edge of the pipes by image pre-processing, and then through the establishment of distance model, the methods efficiently get the pipe centerline. Finally, by analyzing the radial distortion and tangential distortion perspective error , the centerline of detection accuracy is improved. As can be seen from the experimental results, the method we proposed is accurate and reliable and high operating efficiency.

\section{References}

[1] Schaap M, Metz,CT, van Walsum T,et al. Standardized evaluation methodology and reference database for evaluating coronary artery centerline extraction algorithms. Medical Image Analysis. 2009 (5)

[2] Jack Lee,Patricia Beighley,Erik Ritman,Nicolas Smith. Automatic segmentation of 3D micro-CT coronary vascular images. Medical Image Analysis. 2007 (6)

[3] Cemil Kirbas,Francis Quek. A review of vessel extraction techniques and algorithms. ACM Computing Surveys (CSUR) . 2004 (2) 
[3] R.L. Ogniewicz,O. Kübler. Hierarchic Voronoi skeletons. Pattern Recognition. 1995 (3)

[4] Robert Laganière, OpenCV2 Computer Vision Application Programming Cookbook, Birmingham: Packt Publishing Ltd.2011.

[5] Zhang. A Flexible New Technique for Camera Calibration. IEEE Transactions on Pattern Analysis and Machine Intelligence, 22(11):1330-1334, 2000. 